\title{
Time-Varying Copula Models for Longitudinal Data
}

\author{
Dr. Esra Kurum \\ University of California, Riverside, USA \\ esra.kurum@ucr.edu
}

\begin{abstract}
We propose a copula-based joint modeling framework for mixed longitudinal responses. Our approach permits all model parameters to vary with time, and thus will enable researchers to reveal dynamic response-predictor relationships and response-response associations. We call the new class of models timecop because we model dependence using a time-varying copula. We develop a one-step estimation procedure for the timecop parameter vector, and also describe how to estimate standard errors. We investigate the finite sample performance of our procedure via simulation studies, one of which shows that our procedure performs well under ignorable missingness. We also illustrate the applicability of our approach by analyzing binary and continuous responses from the Women's Interagency HIV Study.
\end{abstract}

